\title{
Understanding the financial cost of robotic lobectomy: calculating the value of innovation?
}

\author{
Emily Singer", Peter J. Kneuertz", Desmond M. D’Souza, Susan D. Moffatt-Bruce, Robert E. Merritt \\ Division of Thoracic Surgery, Department of Surgery, The Ohio State University Wexner Medical Center, Columbus, OH, USA \\ \#These authors contributed equally to this work and share first authorship. \\ Correspondence to: Peter J. Kneuertz, MD. Division of Thoracic Surgery, The Ohio State University Wexner Medical Center, 410 West $10^{\text {th }}$ Avenue, \\ N846a Doan Hall, Columbus, OH 43210, USA. Email: Peter.Kneuertz@osumc.edu.
}

\begin{abstract}
Background: The advantages of a minimally-invasive surgical approach compared to conventional open thoracotomy for lung resection have been previously described. While robot-assisted thoracoscopic surgery (RATS) has shown comparable clinical outcomes for lobectomy as compared with video-assisted thoracic surgery (VATS), the cost and inherent value associated with the robotic technology remains a main concern. Methods: We conducted a systematic review of the literature on the cost of RATS lobectomy using studies published prior to December 2017 on MEDLINE and EMBASE.

Results: Six observational studies met our inclusion criteria. Median cost of RATS lobectomy ranged from $\$ 15,440$ to $\$ 22,582$. Operating room (OR) cost was a major contributing factor to overall cost. The lowest per-procedure cost was reported by the highest volume center. Cost definitions were highly variable among studies. The total cost of RATS was similar or lower to open lobectomy, and higher than that of VATS, with cost difference ranging from $\$ 2,901$ to $\$ 4,708$.

Conclusions: Assessment of cost for RATS lobectomy varies significantly. High OR costs may be offset by improved outcomes as compared with open lobectomy, but currently the costs exceed that of VATS lobectomy. Further work is needed to define the cost and actual value parameters for RATS lobectomy.
\end{abstract}

Keywords: Robotic surgery; lobectomy; cost; cost-effectiveness; minimally-invasive

Submitted Jan 10, 2018. Accepted for publication May 29, 2018.

doi: $10.21037 /$ acs.2018.05.18

View this article at: http://dx.doi.org/10.21037/acs.2018.05.18

\section{Introduction}

Posterolateral thoracotomy has been considered the classical and perhaps traditional approach for pulmonary lobectomy (1). Minimally-invasive approaches including video-assisted thoracic surgery (VATS) and more recently robotic-assisted thoracic surgery (RATS) have become increasingly used for lung resection (2). These techniques have proven superior to open thoracotomy with respect to postoperative complications, pain, blood loss, duration of hospitalization, 30-day survival, and have demonstrated comparable long-term outcomes (3-7). While VATS has become widely-accepted, the robotic approach remains a relatively new minimally-invasive approach in the arena of thoracic surgery.
The da Vinci surgical robot (Intuitive Surgical; Sunnvale, CA, USA) was FDA approved in 2000 and has established applications in urologic, gynecologic, and colorectal surgery. The first case-series using the da Vinci for thoracoscopic surgery was reported in 2002 (8). Technical advantages include a 3-dimensional high-definition field of view, tremor filtration, and improved dexterity with the ability to articulate instruments (9). Increasing comparisons of RATS and VATS with respect to surgical outcomes, morbidity, and mortality suggest that the debate about surgical approach is now between these techniques rather than minimally-invasive versus open thoracotomy. In a recent meta-analysis, Emmert et al. conclude that RATS is a non-inferior alternative to VATS for lung resection with regards to perioperative outcomes, and may even 


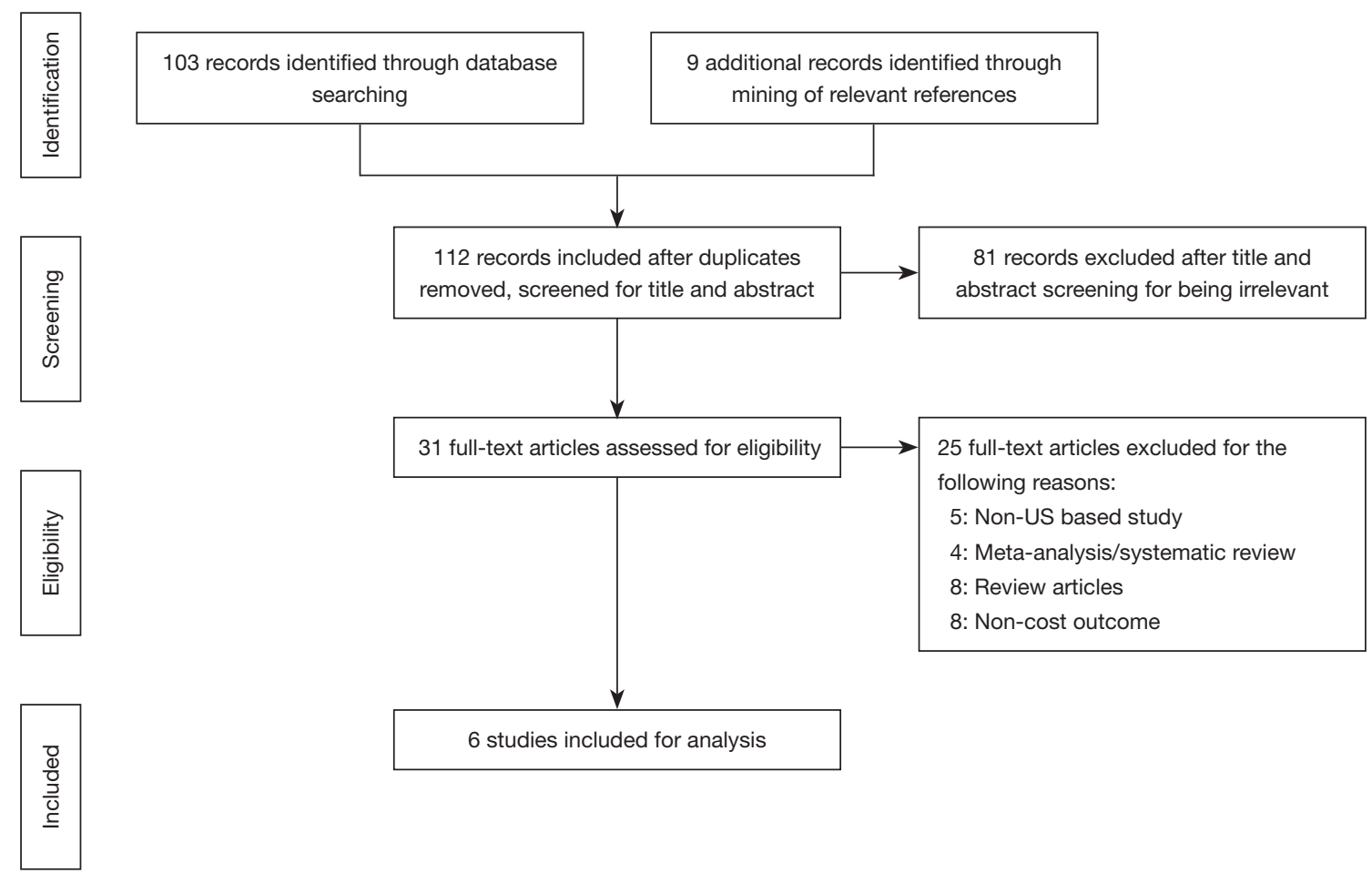

Figure 1 PRISMA diagram of robotic lobectomy cost literature review. PRISMA, preferred reporting items for systematic reviews and meta-analysis.

prove superior with respect to length of stay and chest tube drainage duration (9). Long term survival data are starting to mature as well and show comparable results to VATS and open lobectomy (10). Nonetheless, the high cost of acquiring and maintaining the robot as well as the learning curve for developing surgeon proficiency with this new technology have raised questions about the true value of implementing robotic thoracic surgery programs.

Several studies have sought to delineate the costs of RATS lobectomy. Our aim was to review the current literature, determine cost associated with RATS, compare how costs or value are considered and calculated across studies, and to ultimately examine how costs differ between VATS and open lobectomy. By examining the existing data on cost, we also hope to define common cost parameters that can be employed in future cost effectiveness analyses of robotic lobectomy.

\section{Methods}

\section{Literature search strategy}

A systematic electronic search was performed using the Ovid
MEDLINE and EMBASE databases to identify studies pertinent to the cost of robotic lobectomy. The searches were up to date on December $1^{\text {st }}, 2017$. The following search terms were combined as keywords or MeSH headings: ("cost" or "charges") AND ("robotic" or "robotic surgery" or "robotic assisted" or "RATS") AND ("thoracic surgery" or "thoracic surgical procedure" or "thoracic surgery, video-assisted" or "VATS" or "video-assisted" or "thoracic" or "thoracoscopic" or "lobectomy" or "lobar resection"). Additional sources were mined from reference sections of retrieved studies. Results were not restricted by type, language, or date. As this study did not include experimentation on human or animal subjects, ethical approval was not required for the conduct of this review.

\section{Selection criteria}

The preferred reporting items for systematic reviews and metaanalysis (PRISMA) flow chart is depicted in Figure 1 (11). All studies limited to human subjects and published in English language were deemed eligible for this review if they met the following inclusion criteria: (I) study design: clinical 
trials and observational studies; (II) participants: patients with benign or malignant lung disease; (III) intervention: lobectomy by robotic surgical approach; and (IV) outcome: cost. We excluded studies based on these criteria: (I) study design: abstracts, case reports, conference presentations, expert opinions, reviews, and meta-analysis; (II) participants: patients undergoing sublobar resection; or overlapping patient population between studies; (III) intervention/ comparison: studies that have pooled data for VATS and thoracotomy or studies that compared non-surgical treatment modalities; (IV) outcome: studies that did not include cost as an outcome, or costs at a non-US based institution.

\section{Data extraction and critical appraisal}

Two reviewers (P.J.E. and E.S.) independently extracted information concerning the study design, study period, surgical technique, institution, number of patients, intraoperative parameters (operative time), and postoperative parameters (complications, length of stay, direct cost, indirect cost, total cost). The primary outcome was total cost of lobectomy. Disagreement between the reviewers was resolved by discussion and consensus agreement.

The Downs and Black quality assessment tool was employed to assess the quality of non-randomized studies. This tool consists of 27 questions and is a validated instrument that assesses reporting, internal, and external validity of studies to determine bias (12). We excluded the power assessment (question 27) to score the studies included in the present review. The maximum possible score is 27 . Studies with scores of $0-9$ were considered of low quality, 10-18 of moderate quality, and 19-27 of high quality.

\section{Results}

Initial database query and ancillary reference search yielded 112 results, and after individual review six studies met inclusion criteria (Figure 1). Most results were singleinstitution studies and two were population-based cohort studies (Table 1). Two studies reported on outcomes for individual surgeons $(13,14)$. All studies were of moderate quality based upon the Downs Black scoring system (Table 1). No studies were excluded based on the quality assessment.

\section{Cost definitions}

Cost for a robotic lobectomy is defined as the expense incurred by the institution, hospital, or provider, and can be divided into direct and indirect costs. Cost terminology is summarized in Table 2. The most inclusive description of direct and indirect cost has been described by Nasir et al. (13): direct costs include all items and costs of services in the care of the patient during the hospitalization, for example surgical equipment, the operating room (OR), patient room, lab tests, nursing and ancillary staff, and physical therapy. Indirect cost includes the overhead cost of the building, the amortization of capital equipment and supplies, maintenance of services, utilities and administrative staff. Robot-specific costs can be separated, as outlined by Deen et al. (16): direct costs specific to robotic lobectomy include robotic drapes, disposable instruments, and other supplies required to operate the robot. Indirect cost refers to hospital overhead associated with each surgery, such as building depreciation, salaries of hospital administrators, and hospital services. Robot-specific indirect costs include the purchase price of the robot, which is often reported in the literature as amortized cost, or the total purchase price divided by case volume, as well as capital depreciation and service costs of the robot (16). Total cost is the sum of direct and indirect costs and is distinct from total charges, which is the amount billed to payors (individuals, insurers, Medicare/Medicaid) (13). Data on charges to the payor at the discharge level can be obtained from an individual hospital, and through regional or national payor inpatient care databases, such as the all-payor Nationwide Inpatient Sample (NIS) database (15). Cost-to-charge ratios are provided by the Healthcare Cost and Utilization Project (HCUP) and can be used to indirectly estimate hospital costs through conversion by group-based scaling factors (15). Payment refers to the actual dollar amount received by institutions from payors for the charges associated with each surgery and hospitalization. Of note, robotic surgery has a unique procedure code used for billing. Profit is defined as the difference between payment and cost (13).

\section{Robotic lobectomy cost breakdown}

The included studies span the years 2007-2013 (Table 1). The number of patients who underwent robotic lobectomy in each study ranges from 12 to 282 patients at the University of Alabama, which performed the highest volume of cases among the studies $(13,17)$. While no two studies defined cost in precisely the same way, the lowest total cost of robotic lobectomy was reported by Nasir et al. with median of $\$ 15,440$ (13). This study also reported the shortest OR time (median of 88 minutes), length of stay (median of 2 days), 

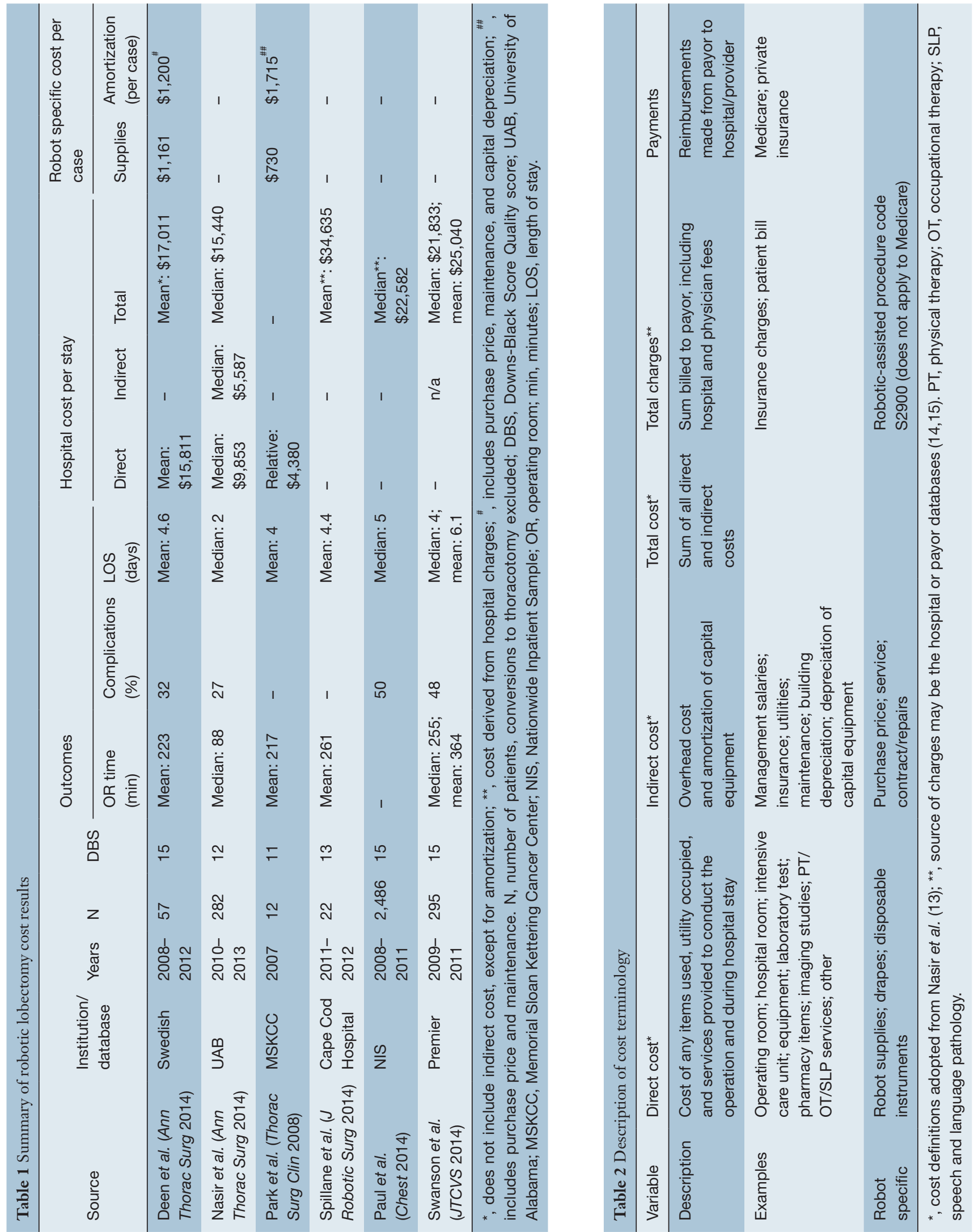


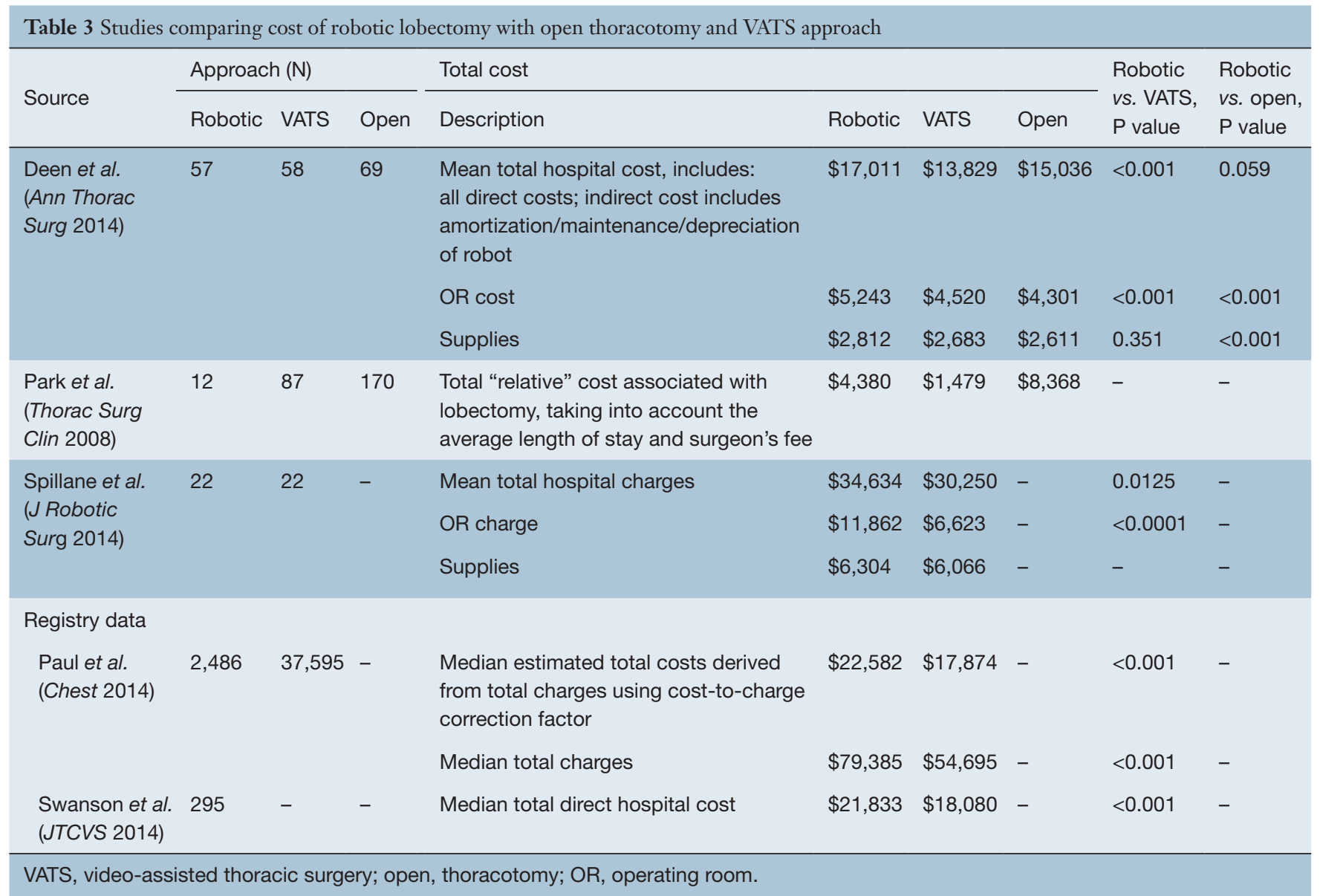

and frequency of complications (27\%). Highest total costs are reported from population-based registry studies. Using the NIS registry data, Paul et al. found median cost of robotic lobectomy to be $\$ 22,582$, while Swanson et al. reported a median cost of $\$ 21,833$ using the Premier registry $(15,18)$. Direct costs are delineated in three of the studies, ranging from $\$ 9,853$ to $\$ 15,811(13,16)$. Only one study included indirect cost in the calculation of total cost, with a median indirect cost per robotic lobectomy of $\$ 5,587$ (13). Deen et al. included an analysis of robot-specific indirect costs, including amortization, depreciation, and maintenance, which totaled $\$ 1,200$ per case (16). Park et al. calculated an added cost of $\$ 1,715$ per case accounting for purchase price and maintenance, but not capital depreciation.

Interestingly, two studies reported hospital charges. At a New England hospital, the mean charge for robotic lobectomy was $\$ 34,635$ (14). In the NIS database study by Paul et al., median charges were $\$ 79,385$ per case (15). Payment data were not included in any study; however, Nasir et al. used the median Medicare reimbursement of $\$ 18,937$ per case to demonstrate that robotic lobectomy can be profitable, reporting a profit margin of $\$ 3,497$ per case (13).

\section{Cost comparison of robotic lobectomy to VATS and open lobectomy}

Five of the six included studies compared the costs and/ or charges of robotic lobectomy to a VATS and/or open thoracotomy approach (Table 3). The total cost of robotic lobectomy was higher than that of VATS in all five, with cost difference ranging from $\$ 2,901$ to $\$ 4,708(15,17)$. Two studies compared the cost of robotic to open lobectomy. Deen et al. showed that RATS was more expensive but not statistically different from open lobectomy, and in the analysis by Park et al. RATS lobectomy was less expensive than open thoracotomy approach by nearly $\$ 4,000(16,17)$. Cost differences between RATS and VATS lobectomy were driven by the expense of the OR itself, with a cost difference 
of $\$ 723(\mathrm{P}<0.001)$, and a charge difference of $\$ 5,238$ $(\mathrm{P}<0.0001)(14,16)$. The cost of supplies was not statistically different between VATS and RATS lobectomy in the study by Deen $e t a l$., and the estimated cost difference of supplies reported by Spillane et al. was an additional \$238 per RATS lobectomy without statistical analysis $(14,16)$.

\section{Discussion}

The use of robotic surgery for pulmonary lobectomy has increased significantly over the past several years in the arena of thoracic surgery (2). A recent analysis of the national cancer database (NCDB) showed that RATS approach was used for $9 \%$ of lobectomies for non-small cell lung cancer (NSCLC) in 2012 (19). Amongst RATS nonadopters, high cost associated with the use of the DaVinci robot remains the most cited point of criticism, and may be a major factor limiting more widespread utilization of this technology. Our aim was to review the existing data on the costs associated with RATS lobectomy. In this systematic review, we show that current data are limited. Existing data are derived from retrospective analyses, mostly from single institutions with small case volume. Additionally, comparison of absolute cost numbers between studies is complicated by the variable definitions and methods of cost assessment between studies. However, despite the differences in surgeon experience and cost definitions, a trend can be derived from these studies, specifically that OR costs are a major contributing factor to the overall cost of RATS lobectomy. Using large registry datasets, two studies have concluded that RATS lobectomy is currently more expensive than VATS. Single institution analyses from two centers have shown that RATS lobectomy is comparable or less costly than open lobectomy.

The effect of case volume on outcomes of RATS lobectomy has been documented (20). The studies identified for this review exhibit small numbers with 12 to 282 patients over several years, representing on average fewer than 20 robotic lobectomy cases per year for three of the four single centers. The population-based studies comprised multiple institutions, with unspecified robotic surgery volume at each center. The learning curve for robotic lobectomy has been reported as at least 20 cases, and it is unclear whether the procedures included in these analyses were performed by surgeons who had surpassed this curve (21). Indeed, difference in surgeon experience likely accounts for the differences in procedure times across the studies, with the highest volume surgeons completing robotic lobectomies in a little over one-third of the time reported at other centers. In addition to fixed robotic instrument and supply costs, OR time is a key parameter to consider for improving cost effectiveness. The study by Nasir et al. suggests that RATS lobectomy can be a profitable endeavor once the institution has not only surpassed its learning curve, but has also reached a high level of proficiency and is performing a large volume of cases, thus realizing economies of scale for a robotic thoracic surgery program (13). In addition to major differences in experience levels of the surgeons, cost definitions varied significantly across these studies with no two using the same cost parameters. Most reported direct costs, and one accounted for indirect costs, including both robot and non-robot specific costs. Understandably, indirect costs may vary significantly by institution and region and are less comparable than direct cost. Among the studies that calculated robot-specific indirect costs, the institutions faced very different purchase prices for the robot, 1 million USD versus 2 million USD $(16,17)$. In addition, many hospitals have existing robotics programs in other surgical specialties, which allows for amortization costs to be distributed. From an economic perspective, a contribution margin may be a more meaningful parameter for cost analyses for centers interested in offering a robotic approach. Contribution margin is the difference between total payments and direct cost, representing a surplus that may be used to offset amortization and robot-specific indirect cost for the institution (22).

In the current American healthcare environment, payment strategies are shifting from a fee-for-service to a bundled payment, value-based approach for reimbursement of surgical procedures. As a result, outcomes relative to cost and quality and the inherent value-equation are more heavily scrutinized. Variability in cost associated with VATS lobectomy has been demonstrated by Medbery et al., with incremental increases in costs for various postoperative complications (23). Cardiopulmonary risk factors, such as chronic obstructive pulmonary disease, coronary artery disease, and body mass index were shown to be associated with increased cost following VATS lobectomy. As experience with RATS lobectomy grows, we can expect to that patient selection will become more liberal as well. Higher risk patients are likely underrepresented in current RATS case series and cost analyses despite the fact that this group may actually derive the greatest benefit from a minimally invasive approach (24). The reduction in length of stay and complication rates following RATS lobectomy has the potential to affect cost savings for the hospital. It remains to be determined whether 
RATS lobectomy is cost effective in a high-risk population and whether true value can be achieved.

In the future, we can expect that continued developments and enhancements in robotic technology may have a significant impact on cost. Particularly, the expected arrival of new robotic platforms from other manufacturers may significantly alter the current single-manufacturer market, with increased competition leading to lower prices and the potential for more widespread use of this technology.

\section{Study limitations}

Important limitations to the present systematic review should be acknowledged. The most notable limitation is the difference in definition of cost across studies included in this review, which renders direct comparison of absolute dollar amounts for cost difficult. We excluded the experience of centers located outside of the United States due to the high degree of variability in cost in different healthcare systems. Finally, as the robotic technology has evolved, institutions from earlier studies were probably using earlier versions of the da Vinci robot, which is currently in its fourth generation, the $\mathrm{Xi}$.

\section{Conclusions}

The systematic review presented herein demonstrates that existing cost analyses of RATS lobectomy are derived primarily from early experiences. Hospital costs associated with RATS lobectomy vary significantly among existing studies. Compared to VATS lobectomy, use of robotic technology is currently associated with higher cost. However, with reduced operative times and improved outcomes, results from high volume centers suggest that cost of RATS lobectomy can be significantly reduced, and the procedure has the potential to be value added. As RATS lobectomy is increasing in popularity and technology of the existing and future competing robotic platforms are evolving, further work is needed to delineate the costs associated with robotic thoracic procedures. Parameters that comprise the total costs in future studies should uniformly account for total direct cost and robotic specific indirect cost for hospitals as well as outcome measures that are inherent to patient-centric value derivation.

\section{Acknowledgements}

None.

\section{Footnote}

Conflicts of Interest: The authors have no conflicts of interest to declare.

\section{References}

1. Moffatt SD, Mitchell JD, Whyte RI. Role of video-assisted thoracoscopic surgery and classic thoracotomy in lung cancer management. Curr Opin Pulm Med 2002;8:281-6.

2. Park BJ, Heerdt PM. Minimally invasive surgical techniques in the treatment of lung cancer. Minerva Chir 2009;64:573-88.

3. Sullivan R, Alatise OI, Anderson BO, et al. Global cancer surgery: delivering safe, affordable, and timely cancer surgery. Lancet Oncol 2015;16:1193-224.

4. Falcoz PE, Puyraveau M, Thomas PA, et al. Videoassisted thoracoscopic surgery versus open lobectomy for primary non-small-cell lung cancer: a propensitymatched analysis of outcome from the European Society of Thoracic Surgeon database. Eur J Cardiothorac Surg 2016;49:602-9.

5. Higuchi M, Yaginuma H, Yonechi A, et al. Long-term outcomes after video-assisted thoracic surgery (VATS) lobectomy versus lobectomy via open thoracotomy for clinical stage IA non-small cell lung cancer. J Cardiothorac Surg 2014;9:88.

6. Whitson BA, Groth SS, Duval SJ, et al. Surgery for earlystage non-small cell lung cancer: a systematic review of the video-assisted thoracoscopic surgery versus thoracotomy approaches to lobectomy. Ann Thorac Surg 2008;86:200816; discussion 2016-8.

7. Zhang L, Gao S. Robot-assisted thoracic surgery versus open thoracic surgery for lung cancer: a system review and meta-analysis. Int J Clin Exp Med 2015;8:17804-10.

8. Melfi FM, Menconi GF, Mariani AM, et al. Early experience with robotic technology for thoracoscopic surgery. Eur J Cardiothorac Surg 2002;21:864-8.

9. Emmert A, Straube C, Buentzel J, et al. Robotic versus thoracoscopic lung resection: A systematic review and meta-analysis. Medicine (Baltimore) 2017;96:e7633.

10. Yang HX, Woo KM, Sima CS, et al. Long-term Survival Based on the Surgical Approach to Lobectomy For Clinical Stage I Nonsmall Cell Lung Cancer: Comparison of Robotic, Video-assisted Thoracic Surgery, and Thoracotomy Lobectomy. Ann Surg 2017;265:431-7.

11. Stewart LA, Clarke M, Rovers M, et al. Preferred Reporting Items for Systematic Review and Meta- 
Analyses of individual participant data: the PRISMA-IPD Statement. JAMA 2015;313:1657-65.

12. Downs SH, Black N. The feasibility of creating a checklist for the assessment of the methodological quality both of randomised and non-randomised studies of health care interventions. J Epidemiol Community Health 1998;52:377-84.

13. Nasir BS, Bryant AS, Minnich DJ, et al. Performing robotic lobectomy and segmentectomy: cost, profitability, and outcomes. Ann Thorac Surg 2014;98:203-8; discussion 208-9.

14. Spillane J, Brooks P. Developing a robotic program in thoracic surgery at Cape Cod Hospital. J Robot Surg 2014;8:213-20.

15. Paul S, Jalbert J, Isaacs AJ, et al. Comparative effectiveness of robotic-assisted vs thoracoscopic lobectomy. Chest 2014;146:1505-12.

16. Deen SA, Wilson JL, Wilshire CL, et al. Defining the cost of care for lobectomy and segmentectomy: a comparison of open, video-assisted thoracoscopic, and robotic approaches. Ann Thorac Surg 2014;97:1000-7.

17. Park BJ, Flores RM. Cost comparison of robotic, videoassisted thoracic surgery and thoracotomy approaches to pulmonary lobectomy. Thorac Surg Clin 2008;18:297300 , vii.

18. Swanson SJ, Miller DL, McKenna RJ Jr, et al. Comparing

Cite this article as: Singer E, Kneuertz PJ, D'Souza DM, Moffatt-Bruce SD, Merritt RE. Understanding the financial cost of robotic lobectomy: calculating the value of innovation? Ann Cardiothorac Surg 2019;8(2):194-201. doi: 10.21037/ acs.2018.05.18 robot-assisted thoracic surgical lobectomy with conventional video-assisted thoracic surgical lobectomy and wedge resection: results from a multihospital database (Premier). J Thorac Cardiovasc Surg 2014;147:929-37.

19. Rajaram R, Mohanty S, Bentrem DJ, et al. Nationwide Assessment of Robotic Lobectomy for Non-Small Cell Lung Cancer. Ann Thorac Surg 2017;103:1092-100.

20. Tchouta LN, Park HS, Boffa DJ, et al. Hospital Volume and Outcomes of Robot-Assisted Lobectomies. Chest 2017;151:329-39.

21. Veronesi G. Robotic thoracic surgery: technical considerations and learning curve for pulmonary resection. Thorac Surg Clin 2014;24:135-41, v.

22. Tedesco G, Faggiano FC, Leo E, et al. A comparative cost analysis of robotic-assisted surgery versus laparoscopic surgery and open surgery: the necessity of investing knowledgeably. Surg Endosc 2016;30:5044-51.

23. Medbery RL, Perez SD, Force SD, et al. Video-assisted thoracic surgery lobectomy cost variability: implications for a bundled payment era. Ann Thorac Surg 2014;97:168692; discussion 1692-3.

24. Ceppa DP, Kosinski AS, Berry MF, et al. Thoracoscopic lobectomy has increasing benefit in patients with poor pulmonary function: a Society of Thoracic Surgeons Database analysis. Ann Surg 2012;256:487-93. 